\title{
MAGNETICALLY-DRIVEN ACTUATION USING LIQUID-PHASE POLYMERIZATION (LPP) AND ITS APPLICATION: A PROGRAMMABLE MIXER
}

\author{
A.K. Agarwal ${ }^{1}$, S.S. Sridharamurthy ${ }^{1}$, T.M. Pearce $^{2}$, G.A. Mensing ${ }^{2}$, D.J. Beebe ${ }^{2}$, and H. Jiang ${ }^{1}$ \\ ${ }^{1}$ Department of Electrical and Computer Engineering, University of Wisconsin-Madison \\ ${ }^{2}$ Department of Biomedical Engineering, University of Wisconsin-Madison \\ Madison, Wisconsin 53706
}

\begin{abstract}
IC-derived microelectromechanical systems (MEMS) technologies have advantages in terms of precision and compatibility with standard fabrication processing. Polymer-based microsystems have recently facilitated promising advances in microfluidics. We present a first demonstration of the combination of these two platforms via the construction and testing of various magnetically-driven devices. Our fabrication process uses microfluidic tectonics $(\mu \mathrm{FT})$ to produce the microscale systems. A programmable micromixer is fabricated that is controlled by an autonomous clutch-based mechanism using responsive hydrogels. Based on the environmental parameters, specifically $\mathrm{pH}$, the micromixer is turned on and off despite the constant presence of an external rotating magnetic driving field. Micromixer mixing efficiencies are also investigated as a function of input flowrates.
\end{abstract}

\section{INTRODUCTION}

Integrated circuit (IC) based MEMS technology has been established with a solid foundation. Its ability to provide highly precise structures and compatibility with standard fabrication methods makes it very attractive to anyone working with microstructures. Recently, a surge in polymer-based microsystems has taken off and allowed for rapid advancement in microfluidic technology. In this work, we utilize a fabrication process, microfluidic tectonics $(\mu \mathrm{FT})$, which uses liquid-phase photopolymerization (LPP) to allow for the rapid creation of microcomponents [1].

We present here an approach to fabricating microsystems that leverages advantages of both aforementioned platforms (MEMS and $\mu \mathrm{FT}$ ). This approach provides unique capabilities and can easily integrate existing sophisticated silicon-based microsystems with polymer-based microfluidics. Polymer-based microfluidics makes use of photosensitive polymers that can be patterned without the additional necessity of a cleanroom environment. Also, LPP is a low-temperature process $\left(<100{ }^{\circ} \mathrm{C}\right)$. Unlike lithography requiring spinning/casting of photosensitive materials on entire substrates, LPP occurs at designated areas on the substrate.

In previous work, researchers demonstrated assembled externally-driven magnetic microstirrers [2, 3]. Many responsive hydrogels also exist that are sensitive to various stimuli, including light, temperature, electric fields, and $\mathrm{pH}$ [4]. Previously, $\mathrm{pH}-$ sensitive hydrogels that undergo volumetric changes (swelling in base solutions and shrinking in acidic solutions) have been used to form autonomous valves [5].

We present a first demonstration via the construction and testing of various magnetically-driven devices, specifically a programmable micromixer. Fabrication of microcomponent devices in situ (inside the channel) and their programmable operation via local fluid parameters are shown here.
FABRICATION METHODS

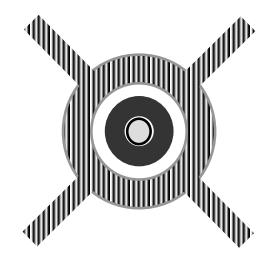

(a)

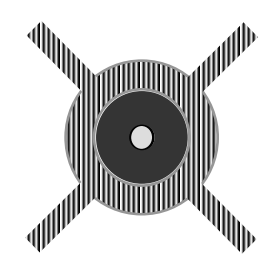

(b)

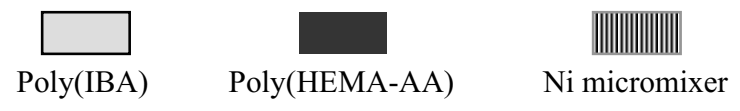

Figure 1. Conceptual diagram of using poly(HEMA-AA) [4] to control the actuation of the Ni micromixer. (a) Low pH causes the hydrogel to shrink, allowing the micromixer to rotate freely. (b) High $\mathrm{pH}$ causes the hydrogel ring to expand in volume, constricting the micromixer's rotational movement.

Magnetically-driven structures are fabricated using polymerbased microfluidics and nickel (Ni) electroplating.

\section{Polymers}

The polymer structures are formed using a liquid IBA-based pre-polymer and (responsive) hydrogels, both which are designed to be sensitive to ultraviolet (UV) light.

The pre-polymer solution consists of three constituents: isobornylacrylate (IBA), tetraethylene glycol dimethacrylate (TeGDMA), and 2,2-dimethoxy-2-phenylacetophenone (DMPA). IBA is the monomer; TeGDMA is the crosslinker; and DMPA is the photoinitiator. Exposure to a UV source causes the prepolymer to harden (poly(IBA)) because of the photoinitiator.

Here, we use $\mathrm{pH}$-sensitive hydrogels to control the actuation of micromixers (see Figure 1). This gel consists of four components: acrylic acid (AA), hydroxy ethyl methacrylate (HEMA), ethylene glycol dimethacrylate (EGDMA), and 2,2dimethoxy-2-phenylacetophenone (DMPA). Exposure to UV light renders a gel matrix that changes its volume configuration depending on its surrounding $(\mathrm{pH})$ environment.

\section{Fabrication}

Here, devices are fabricated on pre-cleaned microscope glass slides $(76.2 \mathrm{~mm} \times 25.4 \mathrm{~mm} \times 1 \mathrm{~mm})$ that have been coated with $\mathrm{Ti} / \mathrm{Cu} / \mathrm{Ti}(0.05 / 0.35 / 0.05 \mu \mathrm{m})$ using a DC sputterer. The bottom and top Ti-layers serve to promote adhesion to the glass and prevent oxidation of the underlying $\mathrm{Cu}$-layer, respectively. The $\mathrm{Cu}$-layer serves as the seed layer for future Ni electroplating. High resolution transparency masks $(3600 \mathrm{dpi})$ are used to pattern the poly(IBA) structures. See Figure 2 for the process flow.

First, a poly(IBA) mold is formed that will serve as a masking layer during the Ni electroplating. A cavity is created between the glass slide and the transparency mask using $125-\mu \mathrm{m}$ 


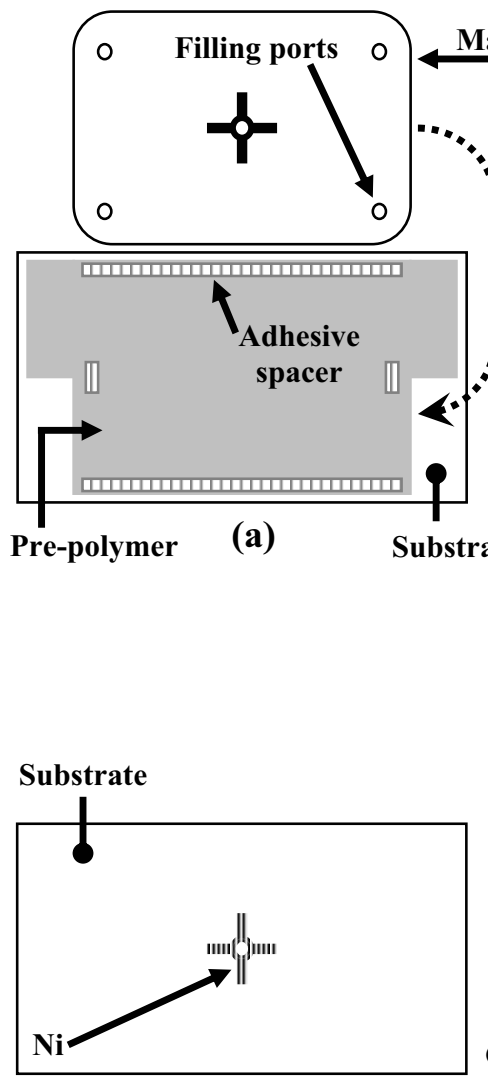

(d)

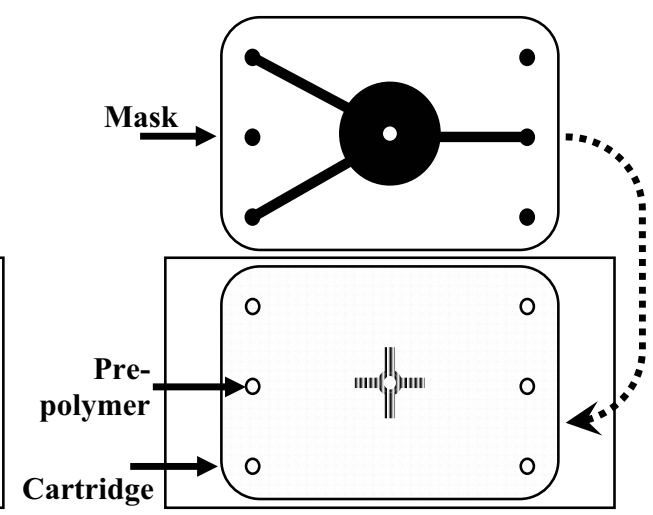

(e)

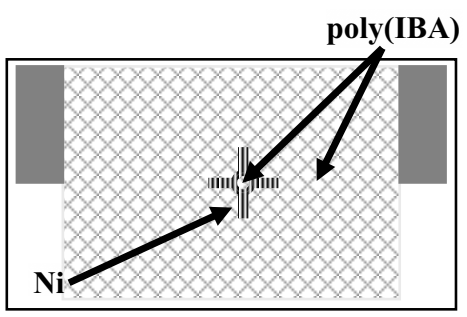

(c)

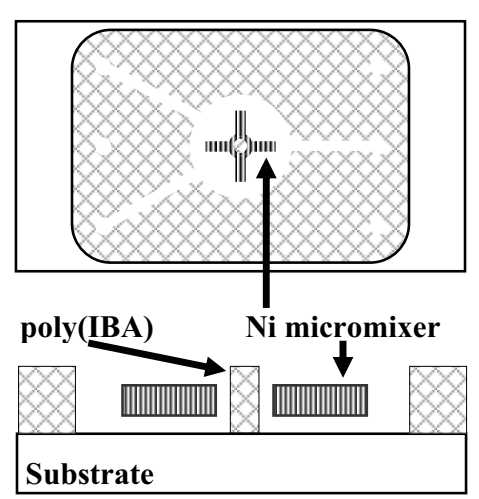

(f)

Figure 2. General process flow for fabricating Ni-based devices (top view). (a) A cavity created between the substrate (coated with $\mathrm{Ti} / \mathrm{Cu} / \mathrm{Ti}$ ) and high resolution transparency mask is filled with the pre-polymer mixture and exposed to UV light. (b) The top Ti-layer is removed, exposing the underlying Cu-layer. (c) Ni electroplating is performed with the poly(IBA) serving as the mold [6]. (d) The poly(IBA) is removed by soaking the device in methanol for several hours. The seed metal layers are also removed [7]. (e) The poly(IBA)based channels, mixing chamber, and post are patterned and realized within a self-containing polycarbonate cartridge. (f) Top and sideview of a functional Ni micromixer post-release with a poly(IBA) post.

thick double-sided adhesive tape. Depending on the desired thickness of the Ni structures, the depth of the cavity varies between $125-375 \mu \mathrm{m}$. The pre-polymer is flowed into the cavity (see Figure 2(a)). The pattern on the mask is transferred to the prepolymer by exposure to UV light (dose ranges from $94 \mathrm{~mJ} / \mathrm{cm}^{2}$ to $131 \mathrm{~mJ} / \mathrm{cm}^{2}$ depending on desired poly(IBA) thickness). The remaining pre-polymer is washed away using ethanol.

Prior to Ni electroplating, the top Ti-layer is removed by placing the glass slide in a bath of $\mathrm{HF}: \mathrm{H}_{2} \mathrm{O}=1: 10$, exposing the underlying $\mathrm{Cu}$-layer (see Figure 2(b)). Once rinsed with $\mathrm{H}_{2} \mathrm{O}$, it is placed in a nickel sulfamate bath $\left(\right.$ Microfab $^{\circledR}$ NI 100, EnthoneOMI, Inc., West Haven, CT) and $\mathrm{Ni}$ is electroplated (current density $=5 \times 10^{-4} \mathrm{~A} / \mathrm{mm}^{2}$ ) [6]. Electroplated Ni thicknesses range from 125-250 $\mu \mathrm{m}$ (see Figure 2(c)) at a rate of approximately $125 \mu \mathrm{m} /$ hour. The poly(IBA) mold is removed by soaking the device in methanol for several hours. The metal seed layers are also removed (see Figure 2(d)). HAC: $\mathrm{H}_{2} \mathrm{O}_{2}: \mathrm{H}_{2} \mathrm{O}=1: 1: 10$ is used to selectively etch $\mathrm{Cu}$ [7].

The channels, fluidic chambers, and post are formed next. A polycarbonate cartridge (HybriWells ${ }^{\mathrm{TM}}$, Grace-Bio Labs, Inc., Bend, OR) with a spacer layer of $250 \mu \mathrm{m}$ is affixed to the glass slide and filled with the pre-polymer using the filling ports (see Figure 2(e)). The pre-polymer is exposed through a transparency mask and subsequently rinsed using ethanol. If so desired, at this step, poly(HEMA-AA) hydrogel posts can be patterned (approximate dose is $680 \mathrm{~mJ} / \mathrm{cm}^{2}$ ) in situ to create a programmable micromixer (see Figure 1). Figure 2(f) shows the final device and cross-section. Access to the channels is accomplished by using plastic pipette tips cut at the end and glued directly onto the polycarbonate channel ports using a general 5-minute epoxy. The Ni structure is finally released by flowing in $\mathrm{Ti}$ and/or $\mathrm{Cu}$-etching solutions.

\section{EXPERIMENTS AND RESULTS}

Experiments were videotaped directly to a computer using a Sony Hyper HAD CCD-Iris/RGB camera (Sony Corp., New York City, NY). An external rotating magnetic stirrer was used to drive the Ni microstructures (Cole Parmer, model 84000-00, Vernon Hills, IL). For controlled and stable fluid flows through the device channels, syringe pumps were used (Cole Parmer, model 74900 series, Vernon Hills, IL).

\section{Experiments and Results}

A programmable micromixer has been developed that is controlled by a clutch-based mechanism using a responsive hydrogel. The autonomous clutch here uses a parameter-sensitive hydrogel ring to control the clutch operation (on and off). It allows individual control of the micromixer despite the constant presence of a rotating external magnetic field (see Figure 1). The micromixer automatically turns on and off based on the local environmental parameter ( $\mathrm{pH}$ for this device), thus providing 
programmable mixing control where the 'program' is 'written' by the fluid in the channel (see Figure 3(a-c)). Figure 3(d) shows the feasibility of fabricating an array of micromixers on a single substrate, all possessing the ability to be individually turned on and off via local environmental parameters that can extend beyond $\mathrm{pH}$, including light, temperature, and electric fields.

The fabrication approach also allows embedding $\mathrm{Ni}$
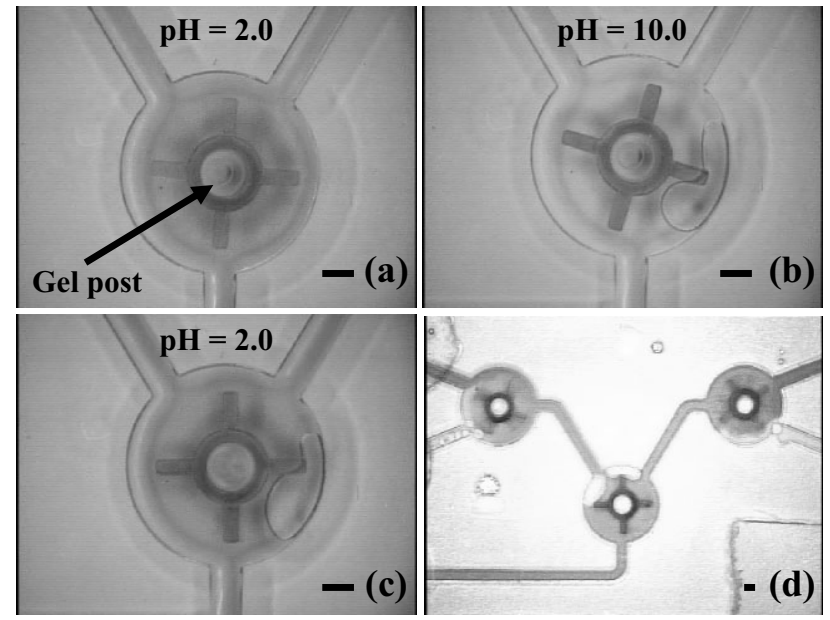

Figure 3. A programmable micromixer that uses a pH-sensitive (poly(HEMA-AA)) hydrogel post $(\phi=1.25 \mathrm{~mm})$. (a) Blue buffer solution of $p H=2.0$ flows through the channel enabling the micromixer to rotate freely $\left(t_{0}\right)$. (b) Yellow buffer solution of $p H=10$ flows through the channel causing the micromixer to stop spinning ( $t_{1}=t_{0}+40$ seconds). (c) Blue buffer solution of $\mathrm{pH}=2.0$ flows through the channel causing the micromixer to rotate again $\left(t_{2}=t_{1}+38\right.$ seconds). (d) A system of micromixers mixing colored solutions. The scale bar represents $0.75 \mathrm{~mm}$.
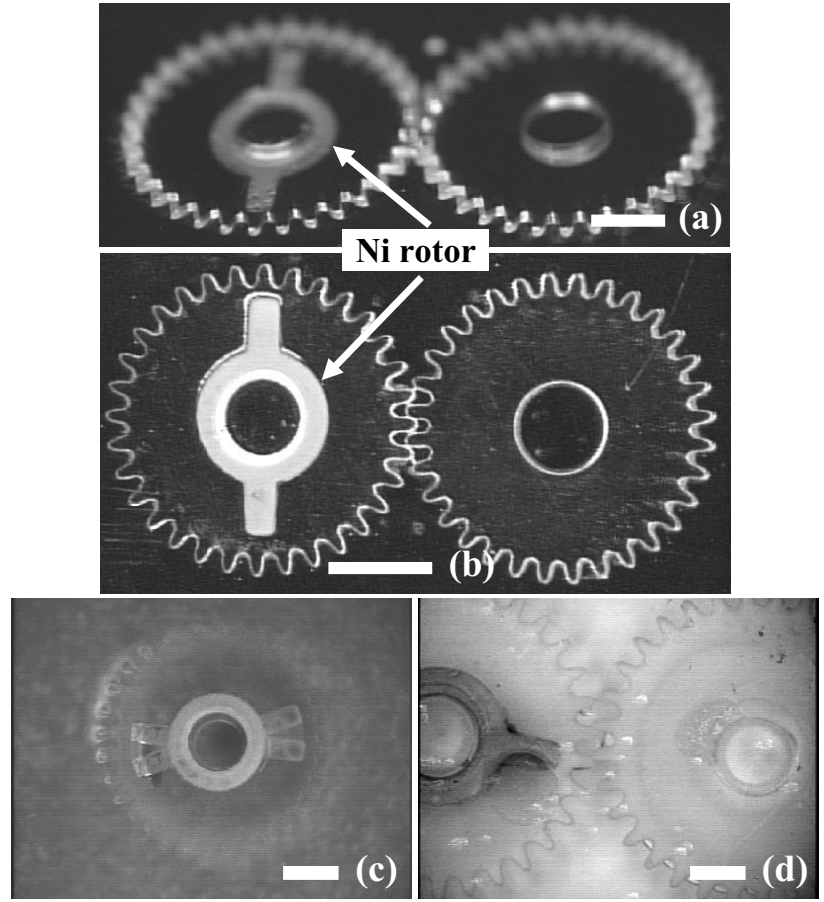

Figure 4. Gear trains and embedded Ni structures. (a) Angled view of the gear train. (b) Top view of the engaged gear train. (c) A single Ni rotor embedded gear rotating freely around a post (open device with no cartridge). (d) The two gears rotating around their own poly(IBA) posts (open device with no cartridge). The scale bar represents $1.25 \mathrm{~mm}$.
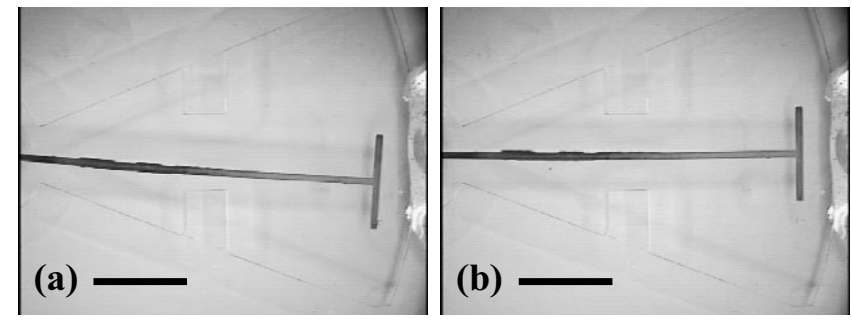

Figure 5. A 20-mm long hammer structure with a 6-mm T-shape at one end, oscillating under the influence of an external magnetic stirrer. (a) The oscillating hammer moving down. (b) The oscillating hammer moving back up (within 1-2 seconds). The scale bar represents $6.0 \mathrm{~mm}$.

structures in free-moving poly(IBA) structures (Figure 4). A Ni rotor $(\sim 125 \mu \mathrm{m}$ tall $)$ is embedded in a $250-\mu \mathrm{m}$ tall poly(IBA) gear. A second poly(IBA) gear is patterned next to the first gear such that the teeth engage with each other (width of each tooth is $\sim 100 \mu \mathrm{m})$. Rotation of the first gear under the influence of an external rotating magnetic stirrer causes the second poly(IBA) gear to rotate in the opposite direction (a gear train). Because the process is liquid-based, the planarization of the poly(IBA) across large topography (i.e., the Ni rotor) is easily realized.

Figure 5 shows a $\mathrm{Ni}$ T-shaped hammer oscillating in a microfluidic channel responding to the rotating magnetic field. The poly(IBA) post (opposite end) cannot be seen in these pictures. The oscillating angle (here, ranging from approximately $2-12^{\circ}$ ) is dependent on the stirrer's RPM, i.e., the higher the RPM, the smaller the angle. With a constant RPM, the hammer's oscillation may also provide an assessment on fluid viscosity. It could also be applied in actuators such as vibromotors [8] or in an on-chip power source with the further integration of a wire coil.

An active micromixer was also fabricated and can be seen mixing two colored solutions in Figure 6 (total flowrate is $16.66 \mu \mathrm{L} / \mathrm{min}$ ). FD\&C Blue 1 and Yellow 5 food coloring dyes are used. The four blades measure $1.25 \mathrm{~mm} \times 0.35 \mathrm{~mm}$ and channel widths are $0.75 \mathrm{~mm}$. Using software NIH Image 1.61 [2], intensity profiles of the cross-section $\sim 3.0 \mathrm{~mm}$ past the mixing chamber were obtained. Mixing efficiencies, shown in Figure 7, are determined for several flowrates. And, since the micromixers are activated by an external rotating magnetic field, there is no need for on-chip power supplies.

This process allows for almost any design of poly(IBA) and Ni structures (see Figure 8). The high resolution transparency masks allow for rapid prototyping and fabrication (electroplating) of Ni structures and liquid-forming of poly(IBA). Thicknesses of these devices range from 100-300 $\mu \mathrm{m}$. Dimensions of the device can range from tens of $\mu \mathrm{m}$ to $\mathrm{mm}$.

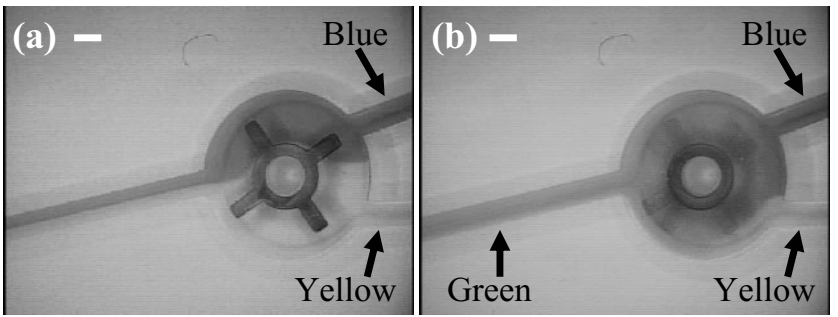

Figure 6. Micromixer mixing blue and yellow solutions. (a) Micromixer at time, $t=0$ second. The two fluids move through the channels in laminar flow without mixing. (b) Magnetic stirrer is activated causing the Ni micromixer to start rotating and mixing the blue and yellow solutions, resulting in a green-colored solution $(t=16$ seconds). The scale bar represents $0.75 \mathrm{~mm}$. 

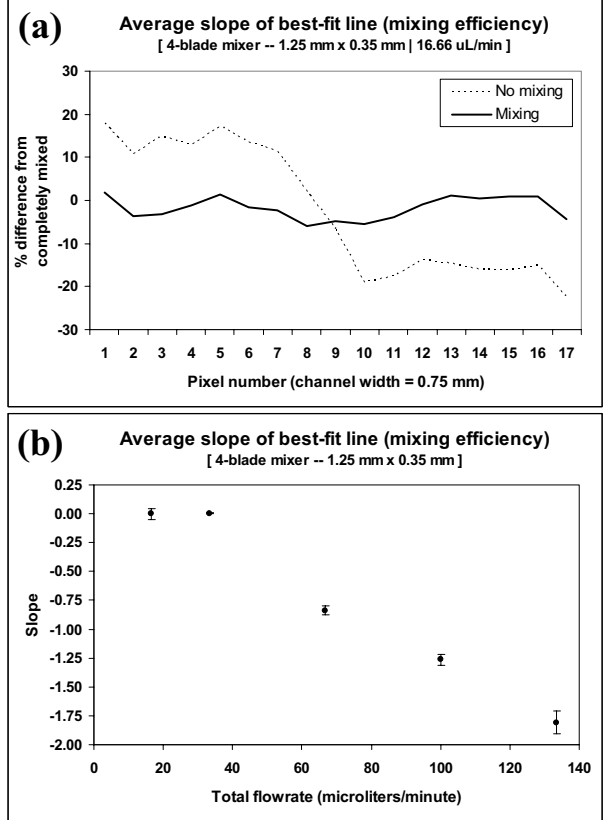

Figure 7. Mixing efficiency plots. (a) Plot of percent difference in intensity between a control mixture, and trials before and after mixing. (b) Average slope as a function of total flowrate (slope $=0$ corresponds to optimal mixing). Vertical bars represent standard deviation.

\section{DISCUSSION}

At the macro scale, the response time of hydrogels to stimuli is very slow since the volumetric change is a diffusion-based process. However, by reducing the scale, the response time can be improved significantly. Because the response is governed by diffusion, the time response improves as the diffusion distance is squared (e.g., a 4-fold reduction in size results in a 16-fold reduction in response time). Thus, these materials are ideally suited to be used at the microscale. Here, instead of patterning a ring of poly(HEMA-AA), smaller and multiple posts may reduce the response time.

Mixing efficiencies depend on many variables, including micromixer dimensions (blade length, width, and thickness), mixing chamber diameter, channel widths, input flowrates, and height of the final poly(IBA) structures. Gear trains also have potential for being incorporated into various systems, including slide rulers, actuators, etc.

The fabrication method can act as a stand-alone process or append to an existing procedure. It allows for extrusion of 2-D structures to form 2.5-D ones, and true 3-dimensional structures
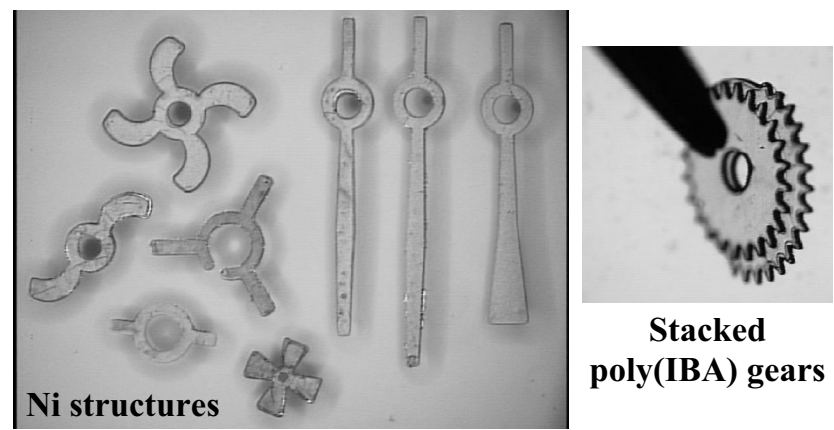

Figure 8. Photograph of multiple Ni structures with different shapes and a two-layer poly(IBA) gear [9]. can also be formed using stereo-lithography. Other substrates such as $\mathrm{Si}, \mathrm{SiN}$, and $\mathrm{SiO}_{2}$ have been tested and pose no problems, further expanding the choice of substrates.

While seed-layer deposition in this work took place in the cleanroom, metal-coated substrates can be purchased, allowing all processes to be performed by researchers without access to a cleanroom facility.

\section{CONCLUSION}

Demonstration of a programmable mixer combining ICderived MEMS and polymer technology has been shown here. Using responsive hydrogels, the on/off status of a single micromixer can be controlled using local environmental parameters. Preliminary experiments on mixing efficiency have also been conducted, showing that as total flowrate increases, mixing efficiency decreases.

The fabrication method can act as a stand-alone process or append to an existing procedure. Unlike conventional lithography requiring spinning/casting of photosensitive materials on entire substrates, LPP occurs at designated areas on the substrate. The process can be appended to MEMS structures that have been previously released. Since the process is liquid-based, topography is not a significant concern. Finally, the ability to control component operation via local fluid parameters expands control scheme possibilities while in situ processing simplifies fabrication and eliminates the need for assembly.

\section{ACKNOWLEDGMENTS}

This work is partly supported by the Wisconsin Alumni Research Foundation (WARF). Travel support has been generously provided by the Transducers Research Foundation and by the DARPA MEMS and DARPA BioFlips programs.

\section{REFERENCES}

[1] D.J. Beebe, J. S. Moore, Q. Yu, R.H. Liu, M.L. Kraft, B-H Jo, C. Devadoss (2000). "Microfluidic tectonics: A comprehensive construction platform for microfluidic systems," PNAS, Dec. 5, 97(25): 13488-13493.

[2] G.A. Mensing, T.M. Pearce, M.D. Graham, and D.J. Beebe (2003). "An externally driven magnetic microstirrer," accepted and to be printed in Philosophical transactions: Mathematical, physical, and engineering sciences.

[3] L-H Lu, K.S. Ryu, and C. Liu (2002). "A magnetic microstirrer and array for microfluidic mixing," JMEMS, October, 11(5): 462-469.

[4] D.T. Eddington and D.J. Beebe (2004). "Flow control with hydrogels," Advanced Drug Delivery Reviews, February 10, 56(2): 199-210.

[5] D.J. Beebe, J.S. Moore, J.M. Bauer, Q. Yu, R.H. Liu, C. Devadoss and B-H. Jo (2000). "Functional hydrogel structures for autonomous flow control inside microfluidic channels," Nature, 404: 588-590.

[6] Microfab ${ }^{\circledR}$ NI 100 data sheet, July 2000, Enthone-OMI Inc., West Haven, CT.

[7] J. Zou, C. Liu, J. Schutt-Aine, J. Chen, and S-M Kang (2000). "Development of a wide tuning range MEMS tunable capacitor for wireless communication systems," Proc. IEDM, December 10-13, 403-406.

[8] A.P. Lee, P.B. Ljung, and A.P. Pisano (1992). "Polysilicon micro vibromotors," IEEE MEMS Proc., Germany, Feb. 4-7.

[9] G.A. Mensing, T.M. Pearce, and D.J. Beebe (2004). "An ultra rapid method of creating $3 \mathrm{D}$ channels and microstructures," submitted to JMEMS 